\title{
The Effect of Noninvasive Ventilation Support on COVID-19 Patients and Risk Factors for Invasive Ventilation - A Retrospective and Multicenter Study
}

\begin{abstract}
Aiyuan Zhou, ',* Qing Song, ${ }^{2, *}$ Yating Peng, (1D ${ }^{2}$ Dingding Deng, 3 Xin Liao, ${ }^{4}$ Peng Huang, ${ }^{5}$

Wenlong Liu, ${ }^{6}$ Zhi Xiang, (1D ${ }^{7}$

Qimi Liu, ${ }^{8}$ Mingyan Jiang, ${ }^{9}$

Xiaoying Huang, ${ }^{10}$ Xudong Xiang, ${ }^{11}$

Hong Peng, (1D ${ }^{2}$ Ping Chen ${ }^{2}$

'Department of Respiratory and Critical Medicine, Xiangya Hospital, Central South University, Changsha, Hunan, 410008, China; ${ }^{2}$ Department of Respiratory and Critical Medicine, The Second Xiangya Hospital, Central South University,

Changsha, Hunan, 4I00II, China; ${ }^{3}$ Department of Respiratory Medicine, The First Attached Hospital of Shaoyang University, Shaoyang, Hunan, 42200I, China; ${ }^{4}$ Department of Respiratory Medicine,

Affiliated Shaoyang Central Hospital of University of South China, Shaoyang, Hunan, 42200I, China;

${ }^{5}$ Department of Respiratory Medicine, Zhuzhou

Central Hospital, Zhuzhou, Hunan, 4I2000, China; ${ }^{6}$ Department of Respiratory Medicine, Yueyang Second People's Hospital, Designated Hospital of Junshan District, Yueyang, Hunan, 4I4005, China; ${ }^{7}$ Department of Respiratory Medicine, The First People's Hospital of Huaihua affiliated to University of South China, Huaihua, Hunan, 4I8000, China; ${ }^{8}$ Department of Respiratory Medicine, The Second People's Hospital of Guilin, Guilin, Guangxi, 54100I, China; 'Department of Respiratory and Critical Medicine, Xiangtan Central Hospital, Xiangtan, Hunan, 4I I I00, China; ${ }^{10}$ Department of Respiratory and Critical Medicine, Loudi Central Hospital, Loudi, Hunan, 4I I 100, China;

"'Department of Emergency Medicine, Second Xiangya Hospital, Central South University,

Changsha, Hunan, 4I00II, China

*These authors contributed equally to this work
\end{abstract}

Correspondence: Ping Chen; Hong Peng Department of Respiratory and Critical Care Medicine, Second Xiangya Hospital, Central South University, I39 Renmin Middle Road, Changsha, Hunan, 4100II, People's Republic of China

Email pingchen0731@csu.edu.cn;

penghong66@csu.edu.cn
Background: Oxygen therapy (OT) is the most widely used supportive regime in patients with hypoxemic acute respiratory failure (ARF) due to severe acute respiratory syndrome coronavirus (SARS-CoV-2) infection. The aim of this study was to identify the effect of noninvasive ventilation support on coronavirus disease 2019 (COVID-19) patients and risk factors for invasive mechanical ventilation (IMV).

Methods: We retrospectively analyzed confirmed COVID-19 subjects from nine hospitals outside Wuhan. All hospitalized patients who tested positive for COVID-19 by real-time polymerase chain reaction between January 1st and March 31st, 2020, were recruited. The patients were divided into four groups based on the most advanced OT regime, including no OT, nasal oxygen therapy, high-flow nasal oxygen therapy (HFNOT) or noninvasive ventilation (NIV), and IMV. Multiple logistic regression models were performed to determine risk factors for IMV.

Results: Of the 683 recruited subjects, 315 (46.1\%) subjects did not need OT, 300 (43.9\%) received nasal oxygen therapy, 51 (7.5\%) received HFNOT or NIV, while $17(2.5 \%)$ subjects had to be intubated. The lactate in the OT group was higher than in the no OT group (2.7 vs 1.6, $P=0.02$ ). In addition, HFNOT or NIV patients had a higher respiratory rate, but a lower $\mathrm{PaO} 2(P<0.001)$. HFNOT and NIV had an obvious beneficial effect on ARF with 75\% of COVID-19 patients recovering from respiratory failure. Patients with IMV were older $(P<0.001)$, had a higher rate of hypertension $(P<0.001)$ and more secondary bacterial infections $(P<0.001)$ compared to those without intubation. The multivariate model showed that secondary bacterial infection $(\mathrm{OR}=6.87$, $P=0.009$ ) was independently associated with IMV failure among COVID-19 patients.

Conclusion: We identified that HFNOT and NIV had an obvious beneficial effect on ARF among COVID-19 patients. We also demonstrated that secondary bacterial infection was an independent risk factor for NIV failure in patients infected by SARS-COV2.

Keywords: COVID-19, SARS-CoV-2, oxygen therapy, invasive mechanical ventilation

\section{Introduction}

In December 2019, a novel coronavirus was identified by the Chinese Center for Disease Control and Prevention (CDC) and was named coronavirus disease 2019 (COVID-19). COVID-19 has since spread rapidly across the globe with rising prevalence and mortality rates. Strategies for the prevention and treatment of COVID-19 are urgently needed. ${ }^{1,2}$

To date, COVID-19 has caused more cases and fatalities than severe acute respiratory syndrome coronavirus (SARS-CoV) and Middle East respiratory syndrome coronavirus (MERS-CoV). ${ }^{3,4}$ Although COVID-19 patients sometimes die 
of shock, multiple organ failure or myocardial damage, ${ }^{5,6}$ respiratory failure is obviously the main cause of mortality, ${ }^{7}$ as reported in previous viral pandemics. ${ }^{8}$ Most affected patients can be supported by noninvasive ventilation (NIV) until the lungs recover. If the situation deteriorates, use of advanced respiratory support, such as invasive mechanical ventilation (IMV) or extracorporeal membrane oxygenation (ECMO) systems are good alternatives to improve hypoxia. So, we speculated that COVID-19 patients may benefit from oxygen therapy in the same way as patients with other types of pneumonia. Notably, in the face of growing numbers of COVID-19 subjects, healthcare providers around the world may benefit from knowing the characteristics of those patients in whom NIV has failed. In addition, the precise risk factors for NIV failure have not been well defined. In this study, we sought to explore characteristics of COVID-19 patients who need of IMV, and particularly to identify risk factors for requiring IMV.

\section{Patients and Methods}

\section{Study Design and Subjects}

We performed a retrospective analysis of data collected from nine COVID-19 designated hospitals outside Wuhan: the Second Xiangya Hospital, the first Attached Hospital of Shaoyang University, the Affiliated Shaoyang Central Hospital of University of South China, Zhuzhou Central Hospital, Yueyang Second People's Hospital, the First People's Hospital of Huaihua affiliated to the University of South China, the Second People's Hospital of Guilin, Xiangtan Central Hospital, and Loudi Central Hospital. This research was approved by the local Ethics Committee of the Second Xiangya Hospital (number: fabh003). All hospitalized patients who tested positive for COVID-19 by real-time polymerase chain reaction between January 1st and March 31st, 2020, were included.

We obtained baseline demographic data and clinical manifestations from a questionnaire designed by the CDC. ${ }^{9}$ The treatment regimens and outcome data were collected from the electronic medical record. The laboratory findings on admission were collected. The data were checked by the doctors in charge and reviewed independently by two researchers. Patients with oxygen therapy (OT) were divided into three groups: ${ }^{10}$ (1) subjects who received nasal oxygen therapy immediately after admission as an initial support strategy then succeeded, named the OT group; (2) subjects who had to receive HFNOT or NIV as a remedy strategy for ARF after the initial regular nasal catheter failed, and then succeeded (HFNOT or NIV group); and (3) patients in whom HFNOT or NIV failed and who received invasive mechanical ventilation (IMV) was administrated as a remedial procedure (IMV group). The date of disease onset was defined as the day when the first symptom was reported. The severity of disease was identified according to the diagnosis and treatment protocol for COVID-19. ${ }^{9}$ The treatment regimen was based on the CDC protocol for COVID-19, which is the official document for COVID-19 management in China. All centers were asked to follow this document. All patients had completed the relevant examinations and treatment plans had been made according to the patient's condition, including the need for HFNOT, NIV and IMV. HFNOT or NIV was applied to subjects who presented with $\mathrm{PaO} 2<60 \mathrm{mmHg}$ or an oxygenation index which is a ratio of $\mathrm{PaO} 2$ to fractional inspired oxygen concentration (FiO2) $(\mathrm{PaO} 2 / \mathrm{FiO} 2)$ of 300 or less despite oxygen delivery through a nasal catheter. $^{9}$ Criteria for IMV included failure to maintain a $\mathrm{PaO} 2 / \mathrm{FiO} 2<300$ despite NIV, inability to protect the airways or to manage copious tracheal secretions, inability to tolerate the face mask, or progression of respiratory failure defined as sustained hypoxemia despite an increase in $\mathrm{FiO} 2$ or the appearance of hypercapnia. ${ }^{9}$ If respiratory distress or hypoxemia did not improve or even worsened within a short time (1-2 hours) following HFNOT or NIV therapy, IMV was performed according to the National Health Commission of the People's Republic of China Chinese management guideline for COVID-19. ${ }^{11}$ Secondary infection was diagnosed if patients had clinical symptoms or signs of nosocomial pneumonia or bacteremia combined with a positive culture of a new pathogen from a lower respiratory tract specimen (including the sputum, transtracheal aspirates, or bronchoalveolar lavage fluid, or from blood samples taken $\geq 48 \mathrm{~h}$ after admission). ${ }^{12}$

\section{Statistical Analysis}

Continuous variables are presented as mean and standard deviation (if data were normally distributed) and median and interquartile range (IQR) values (if data were not normally distributed). Categorical variables were described as frequency rates and percentages. Means for continuous variables were compared by paired $t$-tests or ANOVA test. Proportions of categorical variables were compared using the chi-squared test or Fisher exact test. Adjusted multiple logistic regression models were performed to determine the risk factors for IMV. A value of $P<0.05$ was considered 
statistically significant. All statistical analyses were performed using SPSS version 25.0 software.

\section{Results}

\section{Demographics and Clinical Variables Among COVID-I 9 Subjects}

We identified 683 hospitalized subjects infected by COVID19. The median age of the recruited subjects was 43 years (IQR, 33-55). Hypertension (14.3\%) and diabetes (8.9\%) were the most common comorbidities. Of the 683 subjects, 315 (46.1\%) did not receive OT, 300 (43.9\%) had nasal oxygen therapy, and 68 patients underwent HFNOT or NIV; among these 68 patients, 51 patients recovered successfully from hypoxia, 17 patients had to be intubated, and 4 died (Figure 1). Patients with IMV were older (66 vs 52, $P<$
$0.001)$, had a higher rate of hypertension $(35.3 \%$ vs $29.4 \%$, $P<0.001)$ and more secondary bacterial infections $(58.8 \%$ vs $21.6 \%, P<0.001)$ compared to those who recovered with HFNOT or NIV. Patients who needed IMV had longer hospital stays ( 26 vs 17 days, $P<0.001$ ). Patients who recovered with regular OT received smaller amounts of corticosteroids $(P<0.001)$ and antibiotics $(P<0.001)$ compared to subjects who needed HFNOT, NIV or IMV (Table 1).

\section{Differences in Symptoms and Lab Findings Among COVID-19 Patients without or with Different Kinds of Oxygen Therapy}

Patients with NIV or IMV had a higher rate of dry cough, dyspnea, fever, and fatigue compared to those who did not need oxygen therapy or received nasal oxygen therapy.

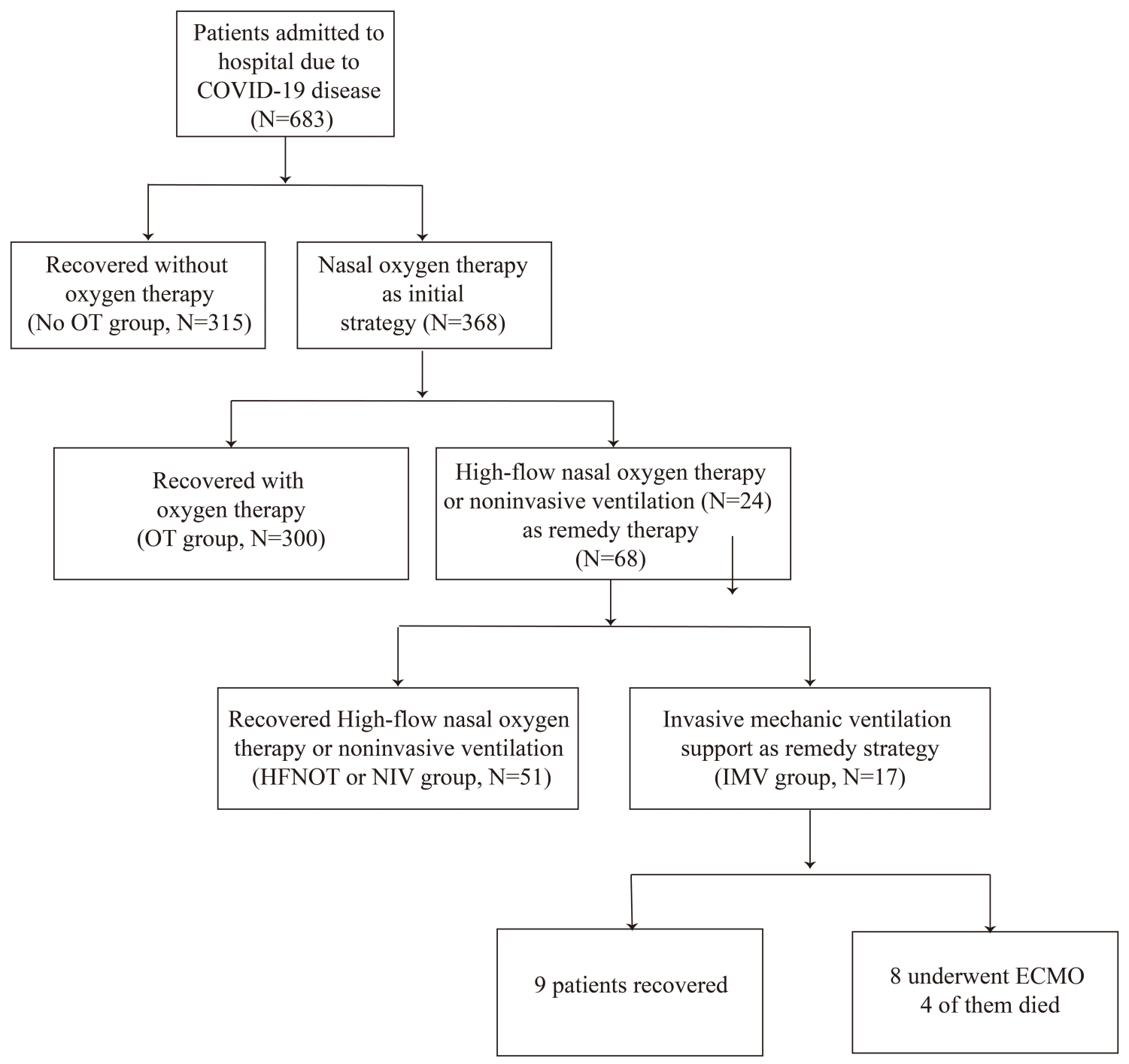

Figure I The flow chart of the study. Of the 683 subjects, 315 (46.1\%) did not receive OT, 300 (43.9\%) had nasal oxygen therapy, 68 patients underwent HFNOT or NIV; among these 68 patients, 51 patients successfully recovered from hypoxia, 17 patients had to be intubated, and 4 patients died. 
Table I Demographics and Clinical Variables Among COVID-19 Subjects

\begin{tabular}{|c|c|c|c|c|c|c|}
\hline Variables & All $(N=683)$ & No OT $(\mathrm{N}=3$ | 5$)$ & OT $(N=300)$ & HFNOT or NIV (N=5I) & IMV $(N=\mid 7)$ & $P^{a}$ \\
\hline Age (IQR) & $43(33-55)$ & $41(30-50)^{\# \alpha^{\wedge}}$ & $45(34-64)^{* 8^{\wedge}}$ & $52(43-64)^{* \#^{\wedge}}$ & $66(54-80)^{* \# \&}$ & $<0.001$ \\
\hline Gender: Female (\%) & $332(48.6)$ & $158(50.2)$ & $149(49.7)$ & $20(39.2)$ & $6(35.3)$ & 0.34 \\
\hline BMI (IQR) & $23(2 \mathrm{I}-26)$ & $23(2 \mathrm{I}-26)$ & $24(22-26)$ & $23(2 \mathrm{I}-25)$ & $23(20-27)$ & 0.55 \\
\hline \multicolumn{7}{|l|}{ Exposure } \\
\hline Contact with Wuhan (\%) & $533(78.0)$ & $257(8 \mathrm{I} .6)$ & $230(76.7)$ & $35(68.6)$ & II (64.7) & 0.26 \\
\hline Comorbidities, any (\%) & $221(32.4)$ & $74(23.5)^{\# \alpha^{\wedge}}$ & $106(35.3)^{* 8^{\wedge}}$ & $29(56.9) * \#$ & $12(70.6)^{* \#}$ & $<0.001$ \\
\hline Diabetes (\%) & $61(8.9)$ & $22(7.0)$ & $28(9.3)$ & $9(17.6)$ & $2(11.8)$ & 0.09 \\
\hline Hypertension (\%) & $98(14.3)$ & $30(9.5)^{\# \varepsilon^{\wedge}}$ & $47(15.7)^{* 8^{\wedge}}$ & $15(29.4)^{* \#^{\wedge}}$ & $6(35.3)^{* \# 8}$ & $<0.001$ \\
\hline Illness onset until hospitalization & $4.0(2-7)$ & $3.0(1-6)^{\#}$ & $3.0(3-8)^{*}$ & $4.0(3-8)$ & $4.0(I-7)$ & 0.003 \\
\hline Shock (\%) & $7(1.0)$ & $0(0)$ & $0(0)$ & $2(3.9)^{* \#}$ & $5(29.4)^{* \# \&}$ & $<0.001$ \\
\hline Hospital days (IQR) & $16(11-23)$ & $16(11-22)^{\wedge}$ & $15(12-23)^{\wedge}$ & $17(12-27)^{\wedge}$ & $26(20-35)^{* \# \&}$ & $<0.001$ \\
\hline Day to recovery (IQR) & $21(16-28)$ & $19(15-25)^{\# \alpha^{\wedge}}$ & $21(16-28) *^{\wedge}$ & $24(|7-3|)^{*}$ & $29(25-37)^{* \#}$ & $<0.001$ \\
\hline Secondary bacterial infection (\%) & $38(5.6)$ & $7(2.2)^{2^{\wedge}}$ & $10(3.3)^{\AA^{\wedge}}$ & $11(21.6)^{* \#^{\wedge}}$ & $10(58.8)^{* \# \&}$ & $<0.001$ \\
\hline Antibiotics (\%) & $326(47.7)$ & $101(32.1)^{\# \alpha^{\wedge}}$ & $164(54.6)^{* \AA^{\wedge}}$ & $45(88.2)^{* \#}$ & $16(94.1)^{* \#}$ & $<0.001$ \\
\hline Corticosteroids (\%) & $149(21.8)$ & $15(4.8)^{\# \alpha^{\wedge}}$ & $80(26.7)^{* 2^{\wedge}}$ & $41(80.4)^{* \#}$ & $13(76.5)^{* \#}$ & $<0.001$ \\
\hline Respiratory rate & $20(20-20)$ & $20(19-20)$ & $20(20-2 I)$ & $20(20-22)^{*} \#^{\wedge}$ & $20(20-20)$ & $<0.001$ \\
\hline
\end{tabular}

Notes: ${ }^{\text {PP }}$ value for statistical difference among the four groups (No OT, OT, HFNOT or NIV, and IMV). *Means statistic difference with No OT group, ${ }^{*}$ Means statistic difference with OT group, ${ }^{8}$ Means statistic difference with HFNOT or NIV group, ${ }^{\wedge}$ Means statistic difference with IMV group.

Abbreviations: COVID-19, coronavirus disease 2019; N, number of subjects; IQR, interquartile range; OT, oxygen therapy; HFNOT, high-flow nasal oxygen therapy; NIV, non-invasive ventilation; IMV, invasive mechanical ventilation.

Patients who recovered with regular OT had a lower level of WBC, neutrophil, platelets, creatine kinase and creatinine relative to those who needed IMV. The patients who failed to recover with HFNOT or NIV had a higher level of WBC, platelets and hemoglobin compared to those in whom hypoxia was corrected with HFNOT or NIV (Tables 2 and 3).

\section{The Effect of HFNOT or NIV on COVID-I9 Patients with Respiratory}

\section{Failure}

Sixty-eight patients underwent HFNOT or NIV, of whom 51 subjects improved while 17 patients deteriorated. Both $\mathrm{PaO} 2$ and the oxygenation index improved substantially after 2 hours of HFNOT or NIV therapy $(88.9 \mathrm{mmHg}$ vs $61.9 \mathrm{mmHg}, P<0.001 ; 372$ vs $225, P<0.001)$. Oxygen saturation also improved after receiving HFNO or NIV therapy $(97.2 \%$ vs $93 \%, P<0.001)$ (Table 4$)$.

\section{Univariate and Stepwise Multivariate Analysis of Risk Factors for the Need of IMV}

Seventeen of 683 subjects in the cohort needed IMV. Univariate analysis showed that several factors were risk factors for the requirement of IMV, including age (odds ratio (OR) $=1.06,95 \%$ confidence interval $(\mathrm{CI})=1.03-1.07, P<0.001)$,
$\mathrm{HBP}(\mathrm{OR}=3.12,95 \% \mathrm{CI}=1.08-9.11, P=0.04)$, secondary bacterial infection $(\mathrm{OR}=31.62,95 \% \mathrm{CI}=9.91-110.92, P<$ $0.001)$, sputum production $(\mathrm{OR}=4.7,95 \% \mathrm{CI}=1.62-13.41$, $P=0.004)$, dyspnea $(\mathrm{OR}=9.77,95 \%, \mathrm{CI}=3.22-29.61, P<$ $0.001)$, fatigue $(\mathrm{OR}=4.85,95 \% \mathrm{CI}=1.62-14.55, P=0.005)$, and neutrophil count $(\mathrm{OR}=1.15,95 \% \mathrm{CI}=1.03-1.28, P=$ $0.01)$. The multivariate model showed that secondary bacterial infection $(\mathrm{OR}=6.87,95 \% \mathrm{CI}=1.63-29.02, P=0.009)$ was independently associated with the need for IMV (Table 5).

\section{Discussion}

The most important finding of our study was that secondary bacterial infection was an independent risk factor for NIV failure among COVID-19 patients, suggesting that clinicians should be alert to bacterial infections, and if necessary, could even consider prophylactic antibiotics. We also found that oxygen therapy had an obvious beneficial effect on ARF due to SARS-COV2 infection.

In this study, we put HFNOT and NIV patients in the same group for analysis, as a study by Grieco et $\mathrm{al}^{13}$ showed that there was no significant difference in the number of days free of respiratory support within 28 days between COVID-19 patients treated with helmet noninvasive ventilation and HFNOT.

Gamberini et al, ${ }^{14}$ who investigated the risk factors associated with the inability to liberate COVID-19 patients 
Table 2 Differences in Symptoms Among COVID-19 Patients without or with Different Kinds of Oxygen Therapy

\begin{tabular}{|c|c|c|c|c|c|c|}
\hline Variables & All $(\mathrm{N}=683)$ & No OT $(\mathrm{N}=3 \mid 5)$ & OT ( $\mathbf{N = 3 0 0 )}$ & HFNOT or NIV $(\mathrm{N}=5 \mathrm{I})$ & IMV (N=I7) & $P^{a}$ \\
\hline Dry cough & $440(64.4)$ & $206(65.4)^{\wedge}$ & $180(60.0)^{\AA^{\wedge}}$ & $39(76.5){ }^{\#}$ & $15(88.2) * \#$ & 0.02 \\
\hline Sputum production & $169(24.7)$ & $92(29.2){ }^{\#}$ & $57(19.0) *^{\wedge}$ & $12(23.5)$ & $8(47.1)^{\#}$ & 0.004 \\
\hline Dyspnea & $101(14.8)$ & $31(9.8)^{\alpha^{\wedge}}$ & $38(12.7)^{8^{\wedge}}$ & $2 I(4 I .2) * \#$ & II (64.7) *\# & $<0.001$ \\
\hline Runny nose & $3(0.4)$ & $3(1.0)$ & $0(0.0)$ & $0(0.0)$ & $0(0.0)$ & 0.32 \\
\hline Sore throat & $39(5.7)$ & $12(3.8) \#$ & $27(9.0) * \&$ & $0(0.0){ }^{\#}$ & $0(0.0)$ & 0.007 \\
\hline Nasal congestion & $15(2.2)$ & $13(4.1)^{\#}$ & $2(0.7) *$ & $0(0.0)$ & $0(0.0)$ & 0.02 \\
\hline Chest tightness & $27(4.0)$ & $10(3.2)$ & II (3.7) & $4(7.8)$ & $2(11.8)$ & 0.15 \\
\hline Palpitation & $3(0.4)$ & $2(0.6)$ & $0(0.0)$ & I (2.0) & $0(0.0)$ & 0.22 \\
\hline Hemoptysis & $26(3.8)$ & $\mathrm{I}(0.3)^{\#^{\wedge}}$ & $21(7.0) *$ & $\mathrm{I}(2.0)^{\wedge}$ & $3(17.6) * \&$ & $<0.001$ \\
\hline Fever & $466(68.2)$ & $199(63.2)^{8^{\wedge}}$ & $206(68.7)^{8^{\wedge}}$ & $44(86.3) * \#$ & $17(100)$ *\# & $<0.001$ \\
\hline Headache & $51(7.5)$ & $21(6.7)^{\&}$ & $21(7.0)^{\&}$ & $8(15.7) * \#$ & I (5.9) & 0.14 \\
\hline Fatigue & $202(29.6)$ & $89(28.3)^{\wedge}$ & $80(27.3)^{\wedge}$ & $20(39.2)$ & II $(64.7) * \#$ & 0.004 \\
\hline Myalgia & $74(10.8)$ & $53(16.8) \# 2$ & $17(5.7) *$ & $3(5.9) *$ & I (5.9) & $<0.001$ \\
\hline Diarrhea & $57(8.3)$ & $27(8.6)$ & $23(7.7)$ & $6(11.8)$ & I (5.9) & 0.77 \\
\hline Nausea & $33(4.8)$ & $8(2.5)^{8^{\wedge}}$ & $17(5.7)$ & $6(11.8) *$ & $2(11.8) *$ & 0.01 \\
\hline Vomit & $8(1.2)$ & $4(1.3)$ & $3(1.0)$ & I (2.0) & $0(0.0)$ & 0.90 \\
\hline
\end{tabular}

Notes: ${ }^{\mathrm{a} P}$ value for statistical difference among the four groups (No OT, OT, HFNOT or NIV, and IMV). *Means statistic difference with No OT group, ${ }^{\#}$ Means statistic difference with OT group, ${ }^{8}$ Means statistic difference with HFNOT or NIV group, ${ }^{\wedge}$ Means statistic difference with IMV group.

Abbreviations: COVID-19, coronavirus disease 2019; N, number of subjects; IQR, interquartile range; OT, oxygen therapy; HFNOT, high-flow nasal oxygen therapy; NIV, non-invasive ventilation; IMV, invasive mechanical ventilation.

Table 3 Differences in Lab Findings Among COVID-19 Patients without or with Different Kinds of Oxygen Therapy

\begin{tabular}{|c|c|c|c|c|c|c|}
\hline Variables & All $(\mathrm{N}=683)$ & No OT $(\mathrm{N}=3 \mid 5)$ & OT $(\mathrm{N}=300)$ & HFNOT or NIV $(\mathrm{N}=5 \mathrm{I})$ & IMV $(N=\mid 7)$ & $P^{a}$ \\
\hline WBC & $4.8(3.7-6.2)$ & $5.0(3.9-6.3)^{\wedge}$ & $4.6(3.6-6.0)^{\wedge}$ & $4.9(4.1-6.0)^{\wedge}$ & $6.7(4.4-9.1) * \# \&$ & 0.009 \\
\hline Lym & $1.2(0.8-1.7)$ & $1.3(0.9-1.7)^{\&}$ & $1.2(0.9-1.6)^{\&}$ & $0.8(0.6-1.2) * \#$ & $0.6(0.4-0.9)$ & 0.02 \\
\hline Neutrophil & $3.0(2.3-4.1)$ & $3.1(2.3-4.1)^{\wedge}$ & $2.9(2.1-3.8)^{\wedge}$ & $3.6(2.8-4.8)$ & $5.2(4.2-7.8)$ *\# & 0.03 \\
\hline Platelets & $196(148-249)$ & $219(165-264){ }^{\# \wedge}$ & $183(143-240) *^{\wedge}$ & $185(|44-2| 3) *^{\wedge}$ & $148(124-165) * \# \&$ & $<0.001$ \\
\hline $\mathrm{Hb}$ & $133(\mid 23-145)$ & $135(126-147){ }^{\#}$ & $130(120-143) * \&$ & $133(124-152) \#^{\#^{\wedge}}$ & $131(116-136)^{\&}$ & 0.005 \\
\hline PT & $12(10-13)$ & $12(11-13){ }^{\#}$ & $12(11-13) *$ & $12(11-13)$ & $12(11-13)$ & 0.002 \\
\hline APTT & $32(29-36)$ & $32(28-36)$ & $33(30-36)$ & $32(26-37)$ & $32(26-38)$ & 0.44 \\
\hline ALT & $2 \mid(|5-3|)$ & $21(15-36){ }^{\#}$ & $21(15-28) * \&$ & $28(|8-4|)^{\#}$ & $23(19-33)$ & 0.005 \\
\hline AST & $24(|9-3|)$ & $23(|8-3|)^{8}$ & $24(|9-3|)^{8}$ & $44(86.3)$ *\# & $29(22-45)$ & 0.009 \\
\hline Albumin & $40(26-44)$ & $42(39-45)^{8^{\wedge}}$ & $39(36-42)$ & $35(33-40) *$ & $33(29-37) *$ & 0.04 \\
\hline Globulin & $26(23-29)$ & $25(19-30){ }^{\# \wedge}$ & $26(23-28) * \&$ & $29(27-31) * \#$ & $27(26-32) *$ & $<0.001$ \\
\hline CK & $68(46-108)$ & $64(45-98)^{\&^{\wedge}}$ & $68(46-106)^{2^{\wedge}}$ & $108(54-195) * \#$ & $168(81-240) * \#$ & $<0.001$ \\
\hline Creatinine & $63(51-76)$ & $65(55-77)^{\wedge}$ & $58(45-72)^{\wedge}$ & $65(55-80)$ & $67(55-87)$ *\# & 0.01 \\
\hline BUN & $4.1(3.2-5.1)$ & $3.8(3.1-4.6)^{8}$ & $4.3(3.2-5.3)^{\&}$ & $5.1(3.8-8.1) * \#$ & $6.5(4.1-8.1)$ & $<0.001$ \\
\hline $\mathrm{LDH}$ & $181(147-224)$ & $180(|54-22|)^{8^{\wedge}}$ & $|7|(|4|-2||)^{8}$ & $24 \mid(188-318)$ *\# & $464(305-511)$ & $<0.001$ \\
\hline Lactate & $1.8(1.2-2.6)$ & $1.6(1.0-2.3)^{\#}$ & 2. $7(1.9-3.1)^{*}$ & $\mathrm{I} .84(\mathrm{I} .25-2.7 \mathrm{I})$ & $2.20(1.05-3.20)$ & 0.02 \\
\hline $\mathrm{PH}$ & $7.5(7.4-7.5)$ & $7.4(7.4-7.5)$ & $7.5(7.4-7.5)$ & $7.5(7.4-7.5)$ & $7.5(7.4-7.5)$ & 0.21 \\
\hline $\mathrm{PaCO} 2$ & $36.7(32.9-4 I . I)$ & 35.1 (28.7-37.7) & $37.5(33.0-41.4)$ & $35.2(29.6-38.7)$ & $33.8(27-88.9)$ & 0.29 \\
\hline $\mathrm{PaO} 2$ & $84.8(72.4-94.6)$ & $94.1(84.3-109) \&^{\wedge}$ & $83.4(71.2-89.4)$ \& & $67.2(48.8-82)^{* \#^{\wedge}}$ & $75(54-97) *$ & $<0.001$ \\
\hline
\end{tabular}

Notes: ${ }^{\mathrm{a} P}$ value for statistical difference among the four groups (No OT, OT, HFNOT or NIV, and IMV). *Means statistic difference with No OT group, ${ }^{\#}$ Means statistic difference with OT group, ${ }^{8}$ Means statistic difference with HFNOT or NIV group, ${ }^{\wedge}$ Means statistic difference with IMV group.

Abbreviations: COVID-19, coronavirus disease 2019; N, number of subjects; IQR, interquartile range; OT, oxygen therapy; HFNOT, High-flow nasal oxygen therapy; NIV, non-invasive ventilation; IMV, invasive mechanical ventilation; WBC, white blood cell; Lym, lymphocyte; Hb, hemoglobin; PT, Prothrombin Time; APTT, activated partial thromboplastin time; ALT; alanine aminotransferase; AST, aspartate aminotransferase; Ck, creatine kinase; BUN, blood urea nitrogen; LDH, Lactate dehydrogenase.

from mechanical ventilation, found that age, sequential organ failure assessment score on intensive care unit admission, respiratory system compliance, $\mathrm{PaO} 2 / \mathrm{FiO} 2$, renal and cardiovascular complications were independent risk factors which was different from our study. Previous studies showed that hypertension was the most common 
Table 4 The Effect of HFNOT or NIV on COVID-19 Patients with Respiratory Failure

\begin{tabular}{|l|c|c|c|}
\hline Variables & $\begin{array}{c}\text { Before HFNOT or } \\
\text { NIV }\end{array}$ & $\begin{array}{c}\text { After HFNOT or } \\
\text { NIV }\end{array}$ & $P$ \\
\hline $\mathrm{PH}$ & $7.4(7.4-7.5)$ & $7.4(7.4-7.5)$ & 0.55 \\
$\mathrm{PaO} 2(\mathrm{mmHg})$ & $61.9(54.6-67.6)$ & $88.9(75.7-97.3)$ & $<0.001$ \\
$\mathrm{PaCO} 2(\mathrm{mmHg})$ & $35.0(31.9-38.0)$ & $37.7(32.5-39.4)$ & 0.05 \\
$\mathrm{SpO} 2 \%)$ & $93.0(91.0-94.0)$ & $97.2(95.8-98.0)$ & $<0.001$ \\
Oxygenation index & $224.9(213.6-244.8)$ & $372.1(337.1-428.8)$ & $<0.001$ \\
\hline
\end{tabular}

Note: Oxygenation index $=\mathrm{PaO} 2 / \mathrm{FiO} 2$.

Abbreviations: COVID-19, coronavirus disease 2019; HFNOT, high-flow nasal oxygen therapy; NIV, non-invasive ventilation; $\mathrm{PaO} 2$, partial artery pressure of oxygen; $\mathrm{PaCO} 2$, partial artery pressure of carbon dioxide; SpO2, oxygen saturation.

comorbidity, and hypertensive patients may be at greater risk of dying from COVID-19. ${ }^{1,15}$ The rate of hypertension in this cohort of patients was low, one of the possible reasons being the younger median age than in previously reported studies (43 vs 51, 49). However, hypertension is not usually an independent risk factor associated with mortality. In our study, we found that patients who needed intubation had higher a rate of hypertension, but we also clarified that hypertension was not an independent risk factor for NIV failure. Additional research is needed to determine whether ACE inhibitors and angiotensin receptor blockers are helpful for patients with COVID-19. We found no difference in diabetes prevalence among patients with different kinds of oxygen therapy.
A previous study showed that among 17 HFNOT patients, 7 (41\%) experienced HFNC failure and received NIV as a rescue therapy. Two (29\%) patients were subsequently intubated after NIV failure. ${ }^{16}$ In our study, 68 patients received HFNOT or NIV and 17 (25\%) of them received IMV as a rescue therapy. The findings of this study revealed that HFNOT had a high success rate, and showed obvious improvement in $\mathrm{PaO} 2$ and oxygenation index, but $25 \%$ of patients treated with HFNOT had to be further intubated. As a result, there is a urgent need to figure out some of the risk factors related to the requirement for IMV. We conducted a multivariate analysis of risk factors for the need of IMV and found that secondary bacterial infection was an independent risk factor for IMV. Physicians should thus be alert to secondary bacterial infection in patients with NIV.

Our study has strengths and limitations. It clearly identified an independent risk factor for the requirement of IMV, providing some suggestions for physicians caring for COVID-19 patients. This was a retrospective study, and although the use of HFNOT or NIV and transition to intubation was based on the Chinese treatment protocol for COVID-19, different physicians have different opinions on the timepoint to switch to intubation. In addition, mortality was not as high as reported for COVID-19 patients in China. In fact, in China, the mortality of COVID-19 in most regions (outside Wuhan) is less than $1 \%,{ }^{17}$ The higher mortality in Wuhan may due to the lack of medical

Table 5 Univariate and Stepwise Multivariate Analysis of Risk Factors for the Need of IMV

\begin{tabular}{|c|c|c|c|c|c|c|}
\hline \multirow[t]{2}{*}{ Variable } & \multicolumn{3}{|c|}{ Univariate } & \multicolumn{3}{|c|}{ Multivariate } \\
\hline & OR & $95 \% \mathrm{Cl}$ & $P$ & OR & $95 \% \mathrm{Cl}$ & $P$ \\
\hline Age (years) & 1.06 & $1.03-1.07$ & 0.001 & 1.03 & $0.98-1.07$ & 0.24 \\
\hline Hypertension & 3.12 & $1.08-9.11$ & 0.04 & 1.50 & $0.33-6.71$ & 0.59 \\
\hline Bacterial infection & 31.62 & $9.91-100.92$ & $<0.001$ & 6.87 & $1.63-29.02$ & 0.009 \\
\hline Dry cough & 3.88 & $0.86-17.47$ & 0.07 & - & - & - \\
\hline Sputum production & 4.70 & $|.62-13.4|$ & 0.004 & 2.53 & $0.64-10.03$ & 0.19 \\
\hline Dyspnea & 9.77 & $3.22-29.6 \mathrm{I}$ & $<0.001$ & 2.79 & $0.59-13.14$ & 0.20 \\
\hline Hemoptysis & 3.76 & $0.99-14.32$ & 0.05 & - & - & - \\
\hline Fever & 5.86 & $1.79-19.2$ & 0.09 & - & - & - \\
\hline Fatigue & 4.85 & $1.62-14.55$ & 0.005 & 2.74 & $0.74-10.19$ & 0.13 \\
\hline White blood cell & 1.17 & $1.03-1.32$ & 0.02 & 1.19 & $0.52-2.70$ & 0.68 \\
\hline Neutrophil & 1.15 & $1.03-1.28$ & 0.01 & 0.93 & $0.40-2.16$ & 0.87 \\
\hline Platelets & 0.99 & $0.98-1.00$ & 0.004 & 0.99 & $0.97-1.00$ & 0.08 \\
\hline Hemoglobin & 0.98 & $0.95-1.01$ & 0.15 & - & - & - \\
\hline Creatine kinase & 1.00 & $1.00-1.01$ & 0.001 & 1.00 & $1.00-1.01$ & 0.22 \\
\hline Creatinine & 1.01 & $1.00-1.01$ & 0.05 & - & - & - \\
\hline
\end{tabular}

Abbreviation: IMV, invasive mechanical ventilation. 
staff, personal protective equipment and ventilators at the early stage of the disease outbreak. In addition, this also may due to the population is relatively young and with few comorbidities in this study when compared with populations more commonly encountered in Western hospitals. ${ }^{18}$

\section{Conclusion}

We identified that HFNOT and NIV had an obvious beneficial effect on ARF among COVID-19 patients. We also demonstrated that secondary bacterial infection was an independent risk factor for NIV failure in patients infected by SARS-COV2.

\section{Abbreviations}

COVID-19, Coronavirus disease 2019; IQR, interquartile range, ICU, intensive care unit; CDC, Chinese Center for Disease Control and Prevention; OT, oxygen therapy; HFNOT, high flow nasal oxygen therapy; NIV, noninvasive ventilation; IMV, invasive mechanical ventilation; SARS-COV-2, severe acute respiratory syndrome coronavirus 2; ARF, acute respiratory failure.

\section{Data Sharing Statement}

The datasets used and/or analysed during the current study are available from the corresponding author on reasonable request.

\section{Ethics Approval}

The research protocol was approved by the local Ethics Committee of the Second Xiangya Hospital of Central South University (number: fabh003) and conducted in accordance with the Declaration of Helsinki and its amendments. Informed consent was waived because of the retrospective nature of the study and the analysis used anonymous clinical data.

\section{Author Contributions}

All authors made a significant contribution to the work reported, whether that is in the conception, study design, execution, acquisition of data, analysis and interpretation, or in all these areas; took part in drafting, revising or critically reviewing the article; gave final approval of the version to be published; have agreed on the journal to which the article has been submitted; and agree to be accountable for all aspects of the work.

\section{Funding}

This work was supported by the National Natural Science Foundation (grant 81770046 and grant 81970044 to Dr Ping Chen).

\section{Disclosure}

None of the authors have a conflict of interest that could affect this manuscript.

\section{References}

1. Huang C, Wang Y, Li X, et al. Clinical features of patients infected with 2019 novel coronavirus in Wuhan, China. Lancet. 2020;395 (10223):497-506. doi:10.1016/S0140-6736(20)30183-5

2. Saidi I, Koumeka PP, Ait Batahar S, Amro L. Factors associated with anxiety and depression among patients with covid-19. Respir Med. 2021;186:106512. doi:10.1016/j.rmed.2021.106512

3. Zhong NS, Zheng BJ, Li YM, et al. Epidemiology and cause of severe acute respiratory syndrome (SARS) in Guangdong, People's Republic of China, in February, 2003. Lancet. 2003;362 (9393):1353-1358. doi:10.1016/S0140-6736(03)14630-2

4. Anderson LJ, Baric RS. Emerging human coronaviruses - disease potential and preparedness. $N$ Engl J Med. 2012;367(19):1850-1852. doi:10.1056/NEJMe1212300

5. Chen N, Zhou M, Dong X, et al. Epidemiological and clinical characteristics of 99 cases of 2019 novel coronavirus pneumonia in Wuhan, China: a descriptive study. Lancet. 2020;395 (10223):507-513. doi:10.1016/S0140-6736(20)30211-7

6. Wang D, Hu B, Hu C, et al. Clinical characteristics of 138 hospitalized patients with 2019 novel coronavirus-infected pneumonia in Wuhan, China. JAMA. 2020;323(11):1061-1069. doi:10.1001/ jama.2020.1585

7. Ruan Q, Yang K, Wang W, Jiang L, Song J. Clinical predictors of mortality due to COVID-19 based on an analysis of data of 150 patients from Wuhan, China. Intensive Care Med. 2020;46 (5):846-848. doi:10.1007/s00134-020-05991-x

8. Matsuyama R, Nishiura H, Kutsuna S, Hayakawa K, Ohmagari N. Clinical determinants of the severity of Middle East respiratory syndrome (MERS): a systematic review and meta-analysis. BMC Public Health. 2016;16(1):1203. doi:10.1186/s12889-016-3881-4

9. National Health Commission of China. New coronavirus pneumonia prevention and control program (5th edn); February 22, 2020. Available from: http://www.gov.cn/zhengce/zhengceku/202002/22/ content_5482010.htm. Accessed September 22, 2021.

10. Rodriguez A, Ferri C, Martin-Loeches I, et al. Risk factors for noninvasive ventilation failure in critically ill subjects with confirmed influenza infection. Respir Care. 2017;62(10):1307-1315. doi: $10.4187 /$ respcare. 05481

11. National health commission of the People's Republic of China Chinese management guideline for COVID-19. Available from: http://www.nhc. gov.cn/yzygj/s7653p/202002/8334a8326dd94d329df351d7da8aefc2/ files/b218cfeb1bc54639af227f922bf6b817.pdf. Accessed September 22, 2021.

12. Garner JS, Jarvis WR, Emori TG, Horan TC, Hughes JM. CDC definitions for nosocomial infections, 1988. Am J Infect Control. 1988;16(3):128-140. doi:10.1016/0196-6553(88)90053-3

13. Grieco DL, Menga LS, Cesarano M, et al. Effect of helmet noninvasive ventilation vs high-flow nasal oxygen on days free of respiratory support in patients with COVID-19 and moderate to severe hypoxemic respiratory failure: the HENIVOT randomized clinical trial. JAMA. 2021;325(17):1731-1743. doi:10.1001/jama.2021.4682 
14. Gamberini L, Tonetti T, Spadaro sshS, et al. Factors influencing liberation from mechanical ventilation in coronavirus disease 2019: multicenter observational study in fifteen Italian ICUs. J Intensive Care. 2020;8(1):80. doi:10.1186/s40560-020-00499-4

15. Wu C, Chen X, Cai Y, et al. Risk factors associated with acute respiratory distress syndrome and death in patients with coronavirus disease 2019 pneumonia in Wuhan, China. JAMA Intern Med. 2020;180(7):934-943. doi:10.1001/jamainternmed.2020.0994

16. Wang K, Zhao W, Li J, Shu W, Duan J. The experience of high-flow nasal cannula in hospitalized patients with 2019 novel coronavirus-infected pneumonia in two hospitals of Chongqing, China. Ann Intensive Care. 2020;10(1):37. doi:10.1186/s13613-02000653-z
17. Cai Q, Huang D, Ou P, et al. COVID-19 in a designated infectious diseases hospital outside Hubei Province, China. Allergy. 2020;75 (7):1742-1752. doi:10.1111/all.14309

18. Ramirez GA, Bozzolo EP, Castelli E, et al. Continuous positive airway pressure and pronation outside the intensive care unit in COVID 19 ARDS. Minerva Med. 2020. doi:10.23736/S00264806.20.06952-9

\section{Publish your work in this journal}

The International Journal of General Medicine is an international, peer-reviewed open-access journal that focuses on general and internal medicine, pathogenesis, epidemiology, diagnosis, monitoring and treatment protocols. The journal is characterized by the rapid reporting of reviews, original research and clinical studies across all disease areas. The manuscript management system is completely online and includes a very quick and fair peer-review system, which is all easy to use. Visit http://www.dovepress.com/ testimonials.php to read real quotes from published authors. 